\title{
ISOSPECTRAL CAYLEY GRAPHS OF SOME FINITE SIMPLE GROUPS
}

\author{
ALEXANDER LUBOTZKY, BETH SAMUELS, AND UZI VISHNE
}

\begin{abstract}
We apply spectral analysis of quotients of the BruhatTits buildings of type $\tilde{A}_{d-1}$ to construct isospectral non-isomorphic Cayley graphs of the finite simple groups $\operatorname{PSL}_{d}\left(\mathbb{F}_{q}\right)$ for every $d \geq 5$ $(d \neq 6)$ and prime power $q>2$.
\end{abstract}

\section{INTRODUCTION}

Let $X_{1}$ and $X_{2}$ be two finite graphs on $n$ vertices, and $A_{i}, i=1,2$, the $n \times n$ adjacency matrix of $X_{i}$. The graphs $X_{1}$ and $X_{2}$ are isospectral (or: cospectral), if the multi-set of eigenvalues of $A_{1}$ is equal to that of $A_{2}$. There are several methods to obtain isospectral graphs, e.g. Seidel switching method [Se], Sunada's method $[\underline{\mathrm{Su}}], \mathrm{Br}],[\mathrm{Lu}]$ and various others. However these methods produce isospectral graphs which are highly non-homogeneous. Very little is known on isospectrality of Cayley graphs. In fact, we are aware only of examples due to Babai $[\mathrm{B}]$, who showed that the dihedral group of order $2 p$ ( $p$ a prime) has at least $p / 64$ pairs of generators which give isospectral (non-isomorphic) Cayley graphs. Since dihedral groups are almost abelian, the question remains for more complicated groups. For example, can one find two sets of generators for the symmetric group $S_{n}$, that will give isospectral non-isomorphic Cayley graphs?

Here we prove the following

Theorem 1. For every $d \geq 5(d \neq 6)$ and every prime power $q$ and $e \geq 1\left(q^{e}>4 d^{2}+1\right)$, there are two systems $A, B$ of generators of the group $\mathrm{PSL}_{d}\left(\mathbb{F}_{q^{e}}\right)$, such that the Cayley graphs Cay $\left(\mathrm{PSL}_{d}\left(\mathbb{F}_{q^{e}}\right) ; A\right)$ and Cay $\left(\mathrm{PSL}_{d}\left(\mathbb{F}_{q^{e}}\right) ; B\right)$ are isospectral and not isomorphic.

Date: Submitted: Dec. 30, 2004. 
The number of generators in $A$ and $B$ can be chosen to be either $r=2 \frac{q^{d}-1}{q-1}$ or $r=\left[\begin{array}{l}d \\ 1\end{array}\right]_{q}+\left[\begin{array}{l}d \\ 2\end{array}\right]_{q}+\cdots+\left[\begin{array}{c}d \\ d-1\end{array}\right]_{q}$ (where $\left[\begin{array}{l}d \\ i\end{array}\right]_{q}$ denotes the number of subspaces of dimension $i$ over $\mathbb{F}_{q}$ in the vector space $\mathbb{F}_{q}^{d}$ ).

Several remarks are in order here. First, our method produces $\varphi(d) / 2$ generating sets for which the Cayley graphs are isomorphic (where $\varphi$ is Euler's function), which explains the exceptional case $d=6$. Secondly, if $A$ is a symmetric set of generators for a group $G$, define the Cayley complex of $G$ with respect to $A$ to be the clique complex induced by the Cayley graph (namely, $g_{0}, \ldots, g_{i} \in G$ form an $i$-cell of the complex iff each pair is connected in the Cayley graph). With this definition, we prove that the Cayley complexes, rather than graphs, are isospectral. Finally, for fixed $d$ and $q$, we obtain infinitely many isospectral pairs which are $r$-regular with the same $r$. This is of its own interest, since the usual methods to construct isospectral $r$-regular graphs give pairs for which $r$ goes to infinity.

Theorem 2. Let $d \geq 3$ and let $r$ be as in Theorem 1, Then, for every $m \in \mathbb{N}$, there exist $m$ isospectral non-isomorphic $r$-regular graphs.

Using Sunada's method, Brooks $[\mathrm{Br}]$ obtained such a result for $r=3$.

Let us now outline our method. The graphs considered in Theorems 1 and 2 are in fact the 1-skeletons of Cayley complexes, or subgraphs of them. The complexes are obtained as quotients of the Bruhat-Tits building $\mathcal{B}_{d}(F)$ associated with the group $\mathrm{PGL}_{d}(F)$, where $F$ is a positive characteristic local field. In spite of the 'finite nature' of the constructed objects, the proof is based on infinite dimensional representation theory and the theory of division algebras over global fields.

In more details, let $F=\mathbb{F}_{q}((t))$ be the field of Laurent power series over a finite field $\mathbb{F}_{q}$ of order $q, G=\operatorname{PGL}_{d}(F)$ and $\mathcal{B}_{d}(F)$ the BruhatTits building associated with $G(c f .[\mathrm{R}],[\mathrm{T}])$. The vertices of $\mathcal{B}=$ $\mathcal{B}_{d}(F)$ can be identified with the cosets $G / K$ where $K=\operatorname{PGL}_{d}(\mathcal{O})$ is a maximal compact subgroup, and $\mathcal{O}=\mathbb{F}_{q}[[t]]$, the ring of integers of $F$. Let $A$ be the adjacency operator or Laplacian acting on $\mathrm{L}^{2}(\mathcal{B})$ (complex functions defined on the vertices). $A$ commutes with the action of $G$, hence it is well defined on $\Gamma \backslash \mathcal{B}$ for every discrete subgroup $\Gamma$ of $G$. In particular, if $\Gamma$ is a uniform lattice in $G$, i.e. a discrete 
cocompact subgroup, $A$ induces on $X=\Gamma \backslash \mathcal{B}$ the adjacency matrix of the 1-skeleton graph, which is an $r$-regular graph for $r=\sum_{i=1}^{d-1}\left[\begin{array}{l}d \\ i\end{array}\right]_{q}$.

Now, $\mathrm{L}^{2}(\Gamma \backslash G)$ is a unitary representation space for $G$. The following is well known (see [Pe and also [LSV1] or [LSV3]):

Proposition 3. Let $G=\mathrm{PGL}_{d}(F), K=\mathrm{PGL}_{d}(\mathcal{O})$ and let $\Gamma_{1}$ and $\Gamma_{2}$ be two discrete cocompact subgroups of $G$. If $\mathrm{L}^{2}\left(\Gamma_{1} \backslash G\right) \cong \mathrm{L}^{2}\left(\Gamma_{2} \backslash G\right)$ as (right) $G$ representation spaces, then the simplicial complexes $\Gamma_{1} \backslash G / K$ and $\Gamma_{1} \backslash G / K$ are isospectral. In fact, they are even strongly isospectral, i.e. with respect to the higher dimensional Laplacians.

Using representation theory (Jacquet-Langlands correspondence) and the theory of division algebras, we prepared in LSV3 various examples of arithmetic subgroups of $\mathrm{PGL}_{d}(F)$ which will provide the means to prove Theorem [2] This is a discrete analog of Theorem 1 of LSV3], claiming that for every $m \in \mathbb{N}$ and $d \geq 3$, the symmetric space $\mathrm{PGL}_{d}(\mathbb{R}) / \mathrm{PO}_{d}(\mathbb{R})$ covers $m$ isospectral non-isomorphic Riemannian manifolds. The proof when translated to our discrete situation, gives $m$ isospectral simplicial complexes (details can be found in LSV3]. Since in our situation the complex is completely determined by its 1-skeleton, we obtain $m$ 1-skeletons which are not isomorphic as graphs. It should be remarked that the proof of isospectrality in LSV3, Theorem 3] uses the Jacquet-Langlands correspondence in positive characteristic; the details of the proof of this correspondence appear in the literature for the characteristic zero case (in general) and for prime $d$ in the characteristic $p$ case. See Remark 1.6 in [LSV1] for more discussion on this.

For the proof of Theorem 11, we use a family of remarkable arithmetic lattices constructed by Cartwright and Steger [CS], which act simply transitively on the vertices of $\mathcal{B}_{d}(F)$. We denote their congruence subgroups by $\Delta(I)$, where $I \triangleleft \mathbb{F}_{q}[t]$ is a prime ideal. We proved in LSV2 that the quotient complexes $\Delta(I) \backslash \mathcal{B}_{d}(F)$ are Cayley complexes for a group which lies between $\mathrm{PGL}_{d}\left(\mathbb{F}_{q^{e}}\right)$ and $\mathrm{PSL}_{d}\left(\mathbb{F}_{q^{e}}\right)$ (here $\left.e=\operatorname{dim}\left(\mathbb{F}_{q}[t] / I\right)\right)$. In particular, their 1-skeletons are Cayley graphs. 
We already have used similar complexes in [LSV2] to give explicit constructions of Ramanujan complexes (see [LSV1]). This time we summon them to provide isospectral Cayley graphs.

The paper is organized as follows: In Section 2 we recall the notation and results from [LSV3] and prove Theorem 2. In Section 3] we recall the Cartwright-Steger lattices and their quotients, as described in [LSV2], and prove Theorem 1. In Section 4 we present explicit examples of isospectral Cayley graphs.

\section{ACKNOWLEDGEMENTS}

The authors acknowledge support of grants by the NSF and the U.S.-Israel Binational Science Foundation.

\section{Proof of Theorem 2}

In LSV3, Theorem 4], we proved the following:

Theorem 4. Let $F$ be a local field of positive characteristic, $G=$ $\mathrm{PGL}_{d}(F)$ where $d \geq 3, K$ a maximal compact subgroup and $\mathcal{B}_{d}(F)=$ $G / K$ the associated Bruhat-Tits building. Then for every $m \in \mathbb{N}$ there exists a family of $m$ torsion free cocompact arithmetic lattices $\left\{\Gamma_{i}\right\}_{i=1, \ldots, m}$ in $G$, such that the finite complexes $\Gamma_{i} \backslash \mathcal{B}_{d}(F)$ are isospectral and not commensurable.

The isospectrality of the $\Gamma_{i} \backslash \mathcal{B}_{d}(F)$ proved in this theorem is 'representation theoretic isospectrality' i.e. $\mathrm{L}^{2}\left(\Gamma_{i} \backslash G\right)$ are isomorphic as $G$-representation spaces, where $G=\operatorname{PGL}_{d}(F)$. As explained there, this implies a 'strong combinatorial isospectrality' in the sense that not only the spectrum of the standard Laplacians is independent of $i$, but the same goes for the spectrum of the higher dimensional or colored Laplacians.

We outline the construction here and refer the reader to [LSV3] for more details. Let $k$ be a global field contained in $F$, and $\nu_{0}$ the valuation of $k$ with respect to which the completion of $k$ is $F$. Since $F \cong \mathbb{F}_{q}((t))$, one may choose $k=\mathbb{F}_{q}(t)$ and $\nu_{0}$ the $t$-adic valuation (i.e. $\nu_{0}\left(t^{i}\right)=i$ and $\nu(f)=0$ for $f \in \mathbb{F}_{q}[t]$ prime to $\left.t\right)$. The completion of $k$ with respect to a valuation $\nu$ is denoted $k_{\nu}$. 
Now let $T$ be a finite set of valuations of $k$ not containing $\nu_{0}$, and let $D_{1}, \ldots, D_{m}$ be central division algebras of degree $d$ over $k$, which are split by $k_{\nu}$ for every valuation $\nu \notin T$ (in particular by $F$ ), and remain division algebras over $k_{\theta}$ for every $\theta \in T$. Moreover we require that $D_{i}$ is not isomorphic as a ring to either $D_{j}$ or $D_{j}^{\text {op }}$ for $i \neq j$ (recall that division algebras over $k$ are uniquely determined - as algebras - by their local invariants, which are in $\{0,1 / d, \ldots,(d-1) / d\})$. We showed in LSV3] that $T$ can be chosen to accommodate $m$ such different division rings if $2^{|T|} /|T|>2 m$.

Let $G_{i}^{\prime}$ denote the algebraic group defined as the multiplicative group $D_{i}^{\times}$modulo its center. Our assumptions guarantee that $G_{i}^{\prime}\left(k_{\theta}\right)$ is a compact group for every $\theta \in T$, and that $G_{i}^{\prime}(F)=\mathrm{PGL}_{d}(F)$. Define the ring

$$
R_{0, T}=\left\{x \in k: \nu(x) \geq 0 \quad \text { for every } \nu \notin T \cup\left\{\nu_{0}\right\}\right\} .
$$

In [LSV3, Section 2] we give an explicit description of $G_{i}^{\prime}$ as an algebraic group defined over $R_{0, T}$. Since $R_{0, T}$ is discrete in the product $\prod_{\nu \in T \cup\left\{\nu_{0}\right\}} k_{\nu}$, it follows that $G_{i}^{\prime}\left(R_{0, T}\right)$ are well defined discrete subgroups of $\mathrm{PGL}_{d}(F)$.

Fix an ideal $I \triangleleft R_{0, T}$, and let $\Gamma_{i}=G_{i}^{\prime}\left(R_{0, T}, I\right)$ be the congruence subgroup (namely the kernel of the map $G_{i}^{\prime}\left(R_{0, T}\right) \rightarrow G_{i}^{\prime}\left(R_{0, T} / I\right)$ induced by the projection $R_{0, T} \rightarrow R_{0, T} / I$ ).

We prove Theorem 4 above in [LSV3], by showing that $\Gamma_{i} \backslash \mathcal{B}_{d}(F)$ are isospectral and non-commensurable (namely they have no finite sheeted common cover). To explain how this result implies Theorem 2. we briefly describe the Bruhat-Tits buildings associated with $G=$ $\mathrm{PGL}_{d}(F)$. Let $\mathcal{O} \subseteq F$ be the valuation ring, then $K=\mathrm{PGL}_{d}(\mathcal{O})$ is a maximal compact subgroup of $G$.

Consider the set of $\mathcal{O}$-submodules of $F^{d}$ of maximal rank (such as $\mathcal{O}^{d}$ ), modulo the equivalence relation $M \sim c M$ for every $c \in F^{\times}$. The action of $\mathrm{GL}_{d}(F)$ on bases of $F^{d}$ induces a transitive action of $G$ on the equivalence classes of modules, with $K$ being the stabilizer of $\left[\mathcal{O}^{d}\right]$. Therefore, we can identify classes of submodules with cosets in $G / K$. To define the graph structure, we take the classes of modules as vertices, and connect two classes $\chi, \chi^{\prime}$ by an edge iff there are representatives 
$M \in \chi$ and $M^{\prime} \in \chi^{\prime}$ such that $t M \subset M^{\prime} \subset M$ (the set of neighbors of a vertex $[M] \in \mathcal{B}^{(0)}$ is in one to one correspondence with the set of vector subspaces of $M / t M \cong \mathbb{F}_{q}^{d}$ over $\mathbb{F}_{q}$, and so the valency is $\left[\begin{array}{l}d \\ 1\end{array}\right]_{q}+$ $\left.\left[\begin{array}{l}d \\ 2\end{array}\right]_{q}+\cdots+\left[\begin{array}{c}d \\ d-1\end{array}\right]_{q}\right)$. The resulting graph is the 1 -skeleton of the building.

For the higher dimensional structure we take the clique complex defined by the 1-skeleton, namely the $i$-cells are the complete subgraphs of size $i+1$. Equivalently, $\chi_{0}, \ldots, \chi_{i}$ form an $i$-cell iff there are representatives $M_{j} \in \chi_{j}$ such that $t M_{0} \subset M_{i} \subset M_{i-1} \subset \cdots \subset M_{1} \subset M_{0}$, after rearrangemenet. In particular there are no $d$-cells. We let $\mathcal{B}_{d}(F)$ denote the resulting complex.

If $\Gamma \subseteq G$ is a cocompact lattice, then the quotient $\Gamma \backslash \mathcal{B}_{d}(F)$ is a finite complex (the complex structure inherited from $\mathcal{B}_{d}(F)$ ). In order for the projection $\mathcal{B}_{d}(F) \rightarrow \Gamma \backslash \mathcal{B}_{d}(F)$ to be a local isomorphism, $\Gamma$ has to be torsion free. However a cocompact lattice of $G$ always has a finite index torsion-free subgroup, and for arithmetic groups we may choose the ideal $I$ small enough so that the $\Gamma_{i}$ are all torsion free.

The complexes $\Gamma_{i} \backslash \mathcal{B}_{d}(F)$ are isospectral by Theorem 4 , so the underlying graphs are also isospectral. On the other hand if two of these graphs are isomorphic, then they define isomorphic clique complexes, which again contradicts Theorem 4. This completes the proof of Theorem 2 for $r=\left[\begin{array}{c}d \\ 1\end{array}\right]_{q}+\left[\begin{array}{c}d \\ 2\end{array}\right]_{q}+\cdots+\left[\begin{array}{c}d \\ d-1\end{array}\right]_{q}$.

The determinant $G / K \rightarrow \mathbb{Z} / d$ induces a $d$-coloring of the (directed) edges: if $t M \subseteq M^{\prime} \subseteq M$, the edge from $M$ to $M^{\prime}$ has color $\operatorname{dim}_{\mathbb{F}_{q}}\left(M / M^{\prime}\right)$. It is easily seen that all edges connected to $[M]$ within one cell, have distinct colors. The colored Laplacian $A_{\ell}, \ell=1, \ldots, d-1$, is then defined on $\mathrm{L}^{2}(G / K)$ by summing over neighbors of color $\ell$, namely $\left(A_{\ell} f\right)(\chi)$ is the sum of $f\left(\chi^{\prime}\right)$ over all neighbors of $\chi$ for which $\left(\chi, \chi^{\prime}\right)$ has color $\ell$. Of course, $A_{1}+\cdots+A_{d-1}$ is the standard Laplacian $A$ of $\mathcal{B}_{d}(F)$. If two quotients $\Gamma_{1} \backslash \mathcal{B}_{d}(F)$ and $\Gamma_{1} \backslash \mathcal{B}_{d}(F)$ of $\mathcal{B}_{d}(F)$ are representationtheoretic isospectral, namely $\mathrm{L}^{2}\left(\Gamma_{1} \backslash G\right) \cong \mathrm{L}^{2}\left(\Gamma_{2} \backslash G\right)$ as $G$-spaces, then they are also isospectral in terms of $A_{\ell}$ for every $\ell$ (see [LSV1, Section 2] for details).

Now let $\mathcal{B}^{1}$ denote the subgraph of $\mathcal{B}_{d}(F)$ defined on all vertices, with edges only of color 1 . From the quotients $\Gamma_{i} \backslash \mathcal{B}^{1}$ we obtain $r$-regular 
graphs for $r=2 \frac{q^{d}-1}{q-1}$, which are isospectral with respect to the standard Laplacian, in this case $A_{1}+A_{d-1}$. On the other hand, in [LSV2, Proposition 2.3] we show that the color 1 part of the graph determines the whole skeleton. Therefore if some quotients of $\mathcal{B}^{1}$ are isomorphic, then so are the respective quotients of $\mathcal{B}_{d}(F)$ which is impossible, by Theorem 4. This completes the proof of Theorem 2

\section{Proof of Theorem 1}

In order to prove Theorem 1 we describe remarkable lattices $\Delta$ in $\mathrm{PGL}_{d}(F)$, which act simply transitively on the vertices of $\mathcal{B}_{d}(F)$. The building can then be identified with the Cayley complex of $\Delta$ with respect to a certain set of generators (where the Cayley complex is the clique complex defined by the Cayley graph). These lattice were introduced in [CS], and used in [LSV2] to give explicit Ramanujan complexes.

We are given a prime power $q$ and an integer $d \geq 2$. The local field $F=\mathbb{F}_{q}((t))$ contains $k=\mathbb{F}_{q}(t)$. Let $\nu_{0}$ denote the $t$-adic valuation, in which $F$ is complete, and let $\mathcal{O}=\mathbb{F}_{q}[[t]]$ be the valuation ring. Let $\sigma$ be a fixed generator of the Galois group of $\mathbb{F}_{q^{d}} / \mathbb{F}_{q}$, extended to $\mathbb{F}_{q^{d}}(t)$ by acting trivially on $t$, and let

$$
D^{(\sigma)}=\mathbb{F}_{q^{d}}(t)\left[z \mid z a=\sigma(a) z, z^{d}=1+t\right]
$$

be a central division algebra of degree $d$ over $k$.

Let $F_{1}=F \otimes_{\mathbb{F}_{q}} \mathbb{F}_{q^{d}}=\mathbb{F}_{q^{d}}((t))$, and note that $F_{1} / F$ is unramified in any valuation of degree 1 of $k$ (i.e. the minus degree valuation, denoted henceforth by $\nu_{1 / t}$, or a valuation induced by a linear prime $p \in \mathbb{F}_{q}[t]$, denoted by $\left.\nu_{p}\right)$. Since $\nu_{0}(1+t)=0$, local class field theory guarantees that $1+t$ is a norm in $F_{1} / F\left[\mathrm{Pi}\right.$, Chapter 17]. But $D^{(\sigma)} \otimes_{k} F=F_{1}[z]$ with $z^{d}=1+t$, so by Wedderburn's criterion [Jac, Corollary 1.7.5], $D^{(\sigma)}$ splits over $F$. There is therefore a natural embedding $D^{(\sigma)} \hookrightarrow \mathrm{M}_{d}(F)$, which we will describe in detail later. As in the previous section, $D^{(\sigma)}$ gives rise to the algebraic group $G^{\prime(\sigma)}$, defined as the multiplicative group of $D^{(\sigma)}$ modulo its center. Then we have $G^{\prime(\sigma)}(k) \hookrightarrow G^{\prime(\sigma)}(F)=$ $\mathrm{PGL}_{d}(F)$. 
Let $\Omega$ denote the set of elements $u\left(1-z^{-1}\right) u^{-1} \in G^{(\sigma)}(k)$, where $u$ runs over $\mathbb{F}_{q^{d}}^{\times} / \mathbb{F}_{q}^{\times}$, and let $\Delta^{(\sigma)}$ be the subgroup of $G^{\prime(\sigma)}(k)$ generated by $\Omega$. It is shown in [LSV2, Proposition 4.9] that the embedding $G^{\prime(\sigma)}(k) \hookrightarrow \mathrm{PGL}_{d}(F)$ takes $\Delta^{(\sigma)}$ into $\mathrm{PGL}_{d}\left(R_{0}\right)$ for

$$
R_{0}=\mathbb{F}_{q}[1 / t]
$$

(and the index $\left[G^{(\sigma)}\left(R_{0}\right): \Delta^{(\sigma)}\right]$ only depends on $q$ and $d$, Proposition 3.5 there). Recall that $K=\mathrm{PGL}_{d}(\mathcal{O})$ is a maximal compact subgroup of $\mathrm{PGL}_{d}(F)$. In [LSV2, Proposition 4.8] we show (following [CS]) that $\Delta^{(\sigma)}$ acts simply transitively on the vertices $\mathrm{PGL}_{d}(F) / K$ of the BruhatTits building, or equivalently that

$$
\Delta^{(\sigma)} \cdot K=\mathrm{PGL}_{d}(F) \quad \text { and } \quad \Delta^{(\sigma)} \cap K=1 .
$$

Therefore we can identify (the vertices of) $\mathcal{B}^{0}=G / K$ with (the elements of) $\Delta^{(\sigma)}$ : every coset $g K$ has a unique representative $\delta K$ with $\delta \in \Delta^{(\sigma)}$.

The complex structure can also be recovered from $\Delta^{(\sigma)}$, as follows. The reduced norm of $1-z^{-1}$ and its conjugates is $t /(1+t)$ LSV2, Proposition 4.1], which is equivalent modulo $\mathcal{O}^{\times}$to the uniformizer $t$. It then follows that the neighbors of color 1 of $\left[\mathcal{O}^{d}\right]$ are $\left[\omega \mathcal{O}^{d}\right]$ for $\omega \in \Omega$. Define $\hat{\Omega}$ to be the set of products $\omega_{1} \ldots \omega_{\ell}$, where $\omega_{1}, \ldots, \omega_{\ell} \in \Omega$, for which there exists $\omega_{\ell+1}, \ldots, \omega_{d} \in \Omega$ such that the product $\omega_{1} \ldots \omega_{\ell} \omega_{\ell+1} \ldots \omega_{d}$ equals 1 (in $G^{\prime(\sigma)}(k)$ ). In Proposition 2.3 and Sections 5 and 6 of LSV2] we show that the neighbors (of arbitrary color) of $\left[g \mathcal{O}^{d}\right]$ are $\left[g \omega \mathcal{O}^{d}\right]$ for $\omega \in \hat{\Omega}$, and that $\mathcal{B}_{d}(F)$ is the Cayley complex of the group $\Delta^{(\sigma)}$ with respect to the generators $\hat{\Omega}$.

The algebras $D^{(\sigma)}$ ramify at exactly two places, $T=\left\{\nu_{1 / t}, \nu_{1+t}\right\}$. The ring defined by Equation (11) in this situation is $R=R_{0, T}=$ $\mathbb{F}_{q}[t, 1 / t, 1 /(1+t)]$. Let $I$ be an ideal of $R$, and set $L=R / I$. Since $\Delta^{(\sigma)} \subseteq G^{(\sigma)}\left(R_{0}\right) \subseteq G^{(\sigma)}(R)$, we can denote by $\Delta^{(\sigma)}(I)$ the kernel of the map $\Delta^{(\sigma)} \rightarrow \mathrm{PGL}_{d}(R / I)$ induced by the projection $\mathrm{PGL}_{d}(R) \rightarrow \mathrm{PGL}_{d}(R / I)$. In [LSV2, Theorem 6.6] we prove that $\Delta_{I}^{(\sigma)}=\Delta^{(\sigma)} / \Delta^{(\sigma)}(I)$ is a subgroup of $\mathrm{PGL}_{d}(L)$ which contains $\mathrm{PSL}_{d}(L)$. Moreover, the index of $\operatorname{PSL}_{d}(L)$ in $\Delta_{I}^{(\sigma)}$ is equal to the order of $t /(1+t)$ in the group $L^{\times} / L^{\times d}$ ([LSV2, Proposition 6.7]), and therefore is independent of $\sigma$. Choosing 
the ideal $I$ properly, we can guarantee that $\Delta_{I}^{(\sigma)}=\operatorname{PSL}_{d}(L)$ (Section 7 of [LSV2]). The explicit embedding of $\Delta_{I}^{(\sigma)}$ into $\mathrm{PGL}_{d}(L)$ is given in LSV2, Section 9]. We elaborate on this in the next section.

To conclude, the set $\hat{\Omega}$ (viewed as elements of $\mathrm{PGL}_{d}(L)$ ) generate a group $\Delta_{I}^{(\sigma)}$ which resides between $\operatorname{PSL}_{d}(L)$ and $\mathrm{PGL}_{d}(L)$, and the resulting Cayley complex is the quotient $\Delta^{(\sigma)}(I) \backslash \mathcal{B}_{d}(F)$.

So far we merely described the Cayley complexes involved. To finish the proof of Theorem 11, we quote again results proved in LSV3]. Let $D_{1}$ and $D_{2}$ be two central division algebras of degree $d$ over $k$, with the same set $T$ of ramification places, but with different invariants at each ramified place (and $\nu_{0} \notin T$ ). Define the algebraic groups $G_{1}^{\prime}$ and $G_{2}^{\prime}$ as in Section 2, For simplicity (and since this is all we need here), assume $|T|=2$. In [LSV3, Theorem 9] we proved that if $D_{2}$ is isomorphic as a ring neither to $D_{1}$ nor to $D_{1}^{\text {op }}$, then the complexes $\Gamma_{1} \backslash \mathcal{B}_{d}(F)$ and $\Gamma_{2} \backslash \mathcal{B}_{d}(F)$ cannot be isomorphic for any finite index torsion-free subgroups $\Gamma_{i} \subseteq G_{i}^{\prime}\left(R_{0, T}\right)$.

The invariants of $D^{(\sigma)}$ defined in Equation (2) are $\frac{-t}{d}, \frac{t}{d}$ where $t$ is chosen so that $\sigma^{t}$ is the Frobenius automorphism on $\mathbb{F}_{q^{d}}$. Assume $\sigma_{1}, \sigma_{2}$ are two generators of $\operatorname{Aut}\left(\mathbb{F}_{q^{d}} / \mathbb{F}_{q}\right)$ such that $\sigma_{2} \neq \sigma_{1}, \sigma_{1}^{-1}$. This situation is possible once $\phi(d)>2$, namely when $d \geq 5, d \neq 6$. Since the local invariants of the algebras $D_{i}=D^{\left(\sigma_{i}\right)}$ defined in Equation (2) are different, Proposition 11 of [LSV3] guarantees that $D_{2}$ is not isomorphic (as a ring) to $D_{1}$, nor to $D_{1}^{\text {op }}$. Since we already know that $\mathcal{B}_{d}(F) \cong \Delta^{\left(\sigma_{i}\right)}$, the above result implies that $\Delta_{I}^{\left(\sigma_{1}\right)}$ and $\Delta_{I}^{\left(\sigma_{2}\right)}$ cannot be isomorphic to each other, when viewed as Cayley complexes with the respective sets of generators $\hat{\Omega}$. But the Cayley complex is completely determined by the Cayley graph, and so $\Delta_{I}^{\left(s_{1}\right)}$ and $\Delta_{I}^{\left(s_{2}\right)}$ cannot be isomorphic as graphs.

In fact, the results of [LSV3] show that as complexes, $\Delta_{I}^{\left(s_{1}\right)}$ and $\Delta_{I}^{\left(s_{2}\right)}$ are also non-commensurable. Of course, they are commensurable as graphs by Leighton's result $[\mathrm{L}$ that every two graphs with the same universal cover have a finite sheeted common cover.

It remains to show that the $\Delta_{I}^{\left(\sigma_{i}\right)}$ are isospectral to each other. Let $\mathcal{V}$ denote the set of valuations of $\mathbb{F}_{q}(t)$, and let $\mathbb{A}$ be ring of adèles over $k$, namely the restricted product of the completions $k_{\nu}$. Also let $\mathbb{A}_{T^{\mathrm{c}}}$ be 
the produce over $\mathcal{V}-T$, and notice that $G_{i}^{\prime}\left(\mathbb{A}_{T^{\mathrm{c}}}\right)=G\left(T^{\mathrm{c}}\right)$ for $i=1,2$, since both $D_{i}$ are split by $k_{\nu}$ for $\nu \in \mathcal{V}-T$.

For a valuation $\nu \in \mathcal{V}$, Let $\mathcal{O}_{\nu}$ denote the valuation ring in the completion $k_{\nu}$, and let $P_{\nu}$ denote the valuation ideal. Set

$$
r_{\nu}=\min \{\nu(a): a \in I\}
$$

and let

$$
U^{(r)}=\prod_{\nu \in \mathcal{V}-\left(T \cup\left\{\nu_{0}\right\}\right)} G\left(\mathcal{O}_{\nu}, P_{\nu}^{r_{\nu}}\right)
$$

an open compact subgroup of $G\left(\mathbb{A}_{T^{\mathrm{c}}-\nu_{0}}\right)$.

Let $J_{i}^{0}$ be the product of $G_{i}^{\prime}\left(k_{\nu_{1+t}}\right)$ and the pre-image of the standard unipotent subgroup of $\mathrm{GL}_{d}\left(\mathbb{F}_{q}\right)$ under the map $G_{i}^{\prime}\left(k_{\nu_{1 / t}}\right) \rightarrow \mathrm{GL}_{d}\left(\mathbb{F}_{q}\right)$ defined by taking the matrices modulo $1 / t$. By Definition 4.7 and Proposition 4.8 in [LSV2], $\Delta^{\sigma_{i}}=G_{i}^{\prime}(k) \cap G\left(\mathbb{A}_{T^{\mathrm{c}}}\right) J_{i}^{0}$, and so

$$
\Delta^{\sigma_{i}}=G_{i}^{\prime}(k) \cap G(F) U^{(r)} J_{i}^{0} .
$$

Since $\left[G_{i}^{\prime}\left(k_{\nu_{1+t}}\right) G_{i}^{\prime}\left(k_{\nu_{1 / t}}\right): J_{i}^{0}\right]$ is independent of $i$, Proposition 15 of LSV3 implies that

$$
\mathrm{L}^{2}\left(\Delta^{\sigma_{1}}(I) \backslash G(F)\right) \cong \mathrm{L}^{2}\left(\Delta^{\sigma_{2}}(I) \backslash G(F)\right)
$$

as $G(F)$-spaces. This implies isospectrality of the complexes

$$
\Delta^{\sigma_{i}}(I) \backslash G(F) / G(\mathcal{O}) \cong \Delta^{\sigma_{i}}(I) \backslash \mathcal{B}_{d}(F)
$$

for $i=1,2$, by [LSV3, Proposition 2]. But $\mathcal{B}_{d}(F) \cong \Delta^{\left(\sigma_{i}\right)}$, so we have the isospectrality of $\Delta^{\left(\sigma_{i}\right)} / \Delta^{\left(\sigma_{i}\right)}(I)$ (again, as Cayley complexes with respect to the generators $\hat{\Omega}$ ). Choosing $I$ suitably (see [LSV2, Section 7]), we can assume $\Delta^{\left(\sigma_{i}\right)} / \Delta^{\left(\sigma_{i}\right)}(I) \cong \operatorname{PSL}_{d}(R / I)$, as asserted.

\section{Explicit Examples of Isospectral Cayley Graphs}

Let $d \geq 5(d \neq 6)$ and let $q$ be a given prime power. In Algorithm 9.2 of [LSV2 we give an explicit construction of Ramanujan Cayley complexes of $\mathrm{PSL}_{d}\left(\mathbb{F}_{q^{e}}\right)$ (see [LSV2, Theorem 7.1]), which is bound to work if $q^{e}>4 d^{2}+1$. (This assumption is only used in LSV2, Proposition 7.3], and can often be ignored. At any rate if $q=2$ then we must take $e>1$ since $\mathbb{F}_{2}[t, 1 / t, 1 /(1+t)]$ has no quotients of dimension 1). Repeating the construction with generators $\sigma_{1}, \sigma_{2}$ of 
$\operatorname{Gal}\left(\mathbb{F}_{q^{d}} / \mathbb{F}_{q}\right)$ such that $\sigma_{2} \neq \sigma_{1}, \sigma_{1}^{-1}$, this realizes the proof of Theorem 1. and provides $\phi(d) / 2$ isospectral non-isomorphic Cayley complexes for any group $\operatorname{PSL}_{d}\left(\mathbb{F}_{q^{e}}\right)$. We illustrate this construction here with $d=5, q=3$ and $e=1$ (which is the smallest case possible).

In order to view $\mathbb{F}_{q^{d}}=\mathbb{F}_{243}$ explicitly, take the irreducible polynomial $\lambda^{5}-\lambda-1$ over $\mathbb{F}_{q}=\mathbb{F}_{3}$. We may write $\mathbb{F}_{243}=\mathbb{F}_{3}\left[t \mid t^{5}=t+1\right]$. We fix the ordered basis $\left\{1, t, t^{2}, t^{3}, t^{4}\right\}$. From now on a linear transformation of $\mathbb{F}_{243}$ (as a vector space over $\mathbb{F}_{3}$ ) is represented via the chosen basis by the $5 \times 5$ matrix. In particular $\mathbb{F}_{243}^{\times}$embeds in $\mathrm{GL}_{5}\left(\mathbb{F}_{3}\right)$ by the regular representation, and the Frobenius automorphism $\phi$, namely exponentiation by 3 , is represented by

$$
\varphi_{1}=\left(\begin{array}{lllll}
1 & 0 & 0 & 1 & 0 \\
0 & 0 & 1 & 1 & 0 \\
0 & 0 & 1 & 0 & 1 \\
0 & 1 & 0 & 0 & 2 \\
0 & 0 & 0 & 1 & 1
\end{array}\right) .
$$

We set $\sigma_{1}=\phi$ and $\sigma_{2}=\phi^{2}$. The representing matrices are $\varphi_{1}$ and $\varphi_{2}=\varphi_{1}^{2}$, respectively.

Define the polynomial $y(\lambda)=(1+\lambda)^{5}-1$ (this amounts to taking $\beta=1$ in Step (1) of [LSV2, Algorithm 9.2]). Take $\alpha=1$, so that $\gamma=y(1)=2^{5}-1=1$ in Step (2) of the Algorithm. The minimal polynomials of these elements are $p(\lambda)=g(\lambda)=\lambda-1$. (Here $g(t)$ must not be invertible in $R_{0, T}$, namely $\left.g(\lambda) \neq \lambda, \lambda+1\right)$. It is possible to construct the Cayley complexes over the local ring $\mathbb{F}_{3}[t] /\left\langle g(t)^{s}\right\rangle$ for every $s \geq 1$. We continue with $s=1$, so in the notation of Step (3) in the Algorithm, $\bar{L}=\mathbb{F}_{3}$ and $x=1$. For $i=1,2$, let $z_{i}$ be the matrix $(1+\beta x) \varphi_{i}=(1+1 \cdot 1) \varphi_{i}=2 \varphi_{i}=-\varphi_{i}\left(\right.$ since $\varphi_{i} a=a^{3^{i}} \varphi_{i}$ for $a \in \mathbb{F}_{243}$, conjugation by $z_{i}$ induces the automorphism $\sigma_{i}$, as in the definition of $D_{i}$ in Equation (2). Moreover $z_{i}^{5}=-\varphi_{i}^{5}=-1=1+\alpha$ ). 
Now we define the elements of $\Omega^{\left(\sigma_{i}\right)}$. Let $b^{(i)}=1-z_{i}^{-1}=1+\varphi_{i}^{4}$, namely

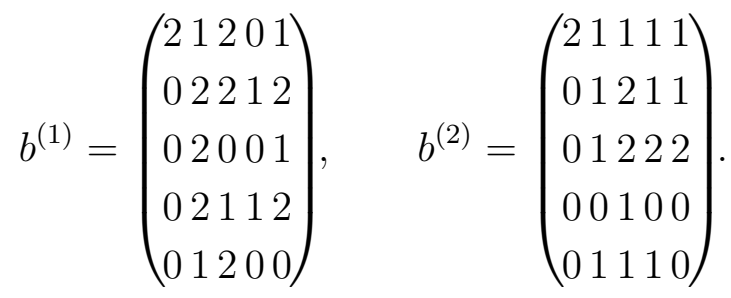

Notice that $\left(b^{(1)}\right)^{3}=\left(1+\varphi_{1}^{4}\right)^{3}=1+\varphi_{1}^{12}=1+\varphi_{1}^{2}=1+\varphi_{2}=b^{(2)}$, and $\left(b^{(2)}\right)^{3}=\left(1+\varphi_{1}^{2}\right)^{3}=1+\varphi_{1}^{6}=b^{(1)}$.

Rather than ranging over all $u \in \mathbb{F}_{243}^{\times} / \mathbb{F}_{3}^{\times}$, we take a generator. One can check that $t^{121}=1$ in $\mathbb{F}_{243}$, while $t^{11}=t^{3}-t^{2}+t \neq 1$. In our basis, multiplication by $t$ corresponds to the matrix

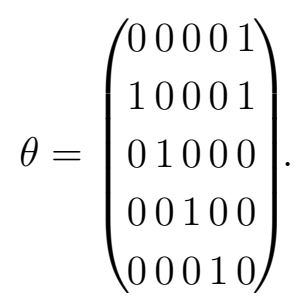

For $i=1,2$ and $j=0, \ldots, 120$, let $b_{j}^{(i)}=\theta^{j} b^{(i)} \theta^{-j}$, and set $\Omega^{\left(\sigma_{i}\right)}=$ $\left\{b_{0}^{(i)}, \ldots, b_{120}^{(i)}\right\}$. Let $\bar{\Omega}^{(i)}$ denote the union of $\Omega^{\left(\sigma_{i}\right)}$ and its inverses. Let $\hat{\Omega}^{(i)}$ denote the products $b_{j_{1}}^{(i)} \ldots b_{j_{k}}^{(i)}, k=1, \ldots, 4$, which can be completed to a product of 5 matrices from $\Omega^{(i)}$ which equals 1 . These products correspond to the $\left[\begin{array}{l}5 \\ 1\end{array}\right]_{3}+\left[\begin{array}{l}5 \\ 2\end{array}\right]_{3}+\left[\begin{array}{l}5 \\ 3\end{array}\right]_{3}+\left[\begin{array}{l}5 \\ 4\end{array}\right]_{3}=121+1210+1210+$ $121=2662$ vector subspaces of $\mathbb{F}_{3}^{5}$, and $\hat{\Omega}^{(i)}$ constitute symmetric sets of generators for $\operatorname{PSL}_{5}\left(\mathbb{F}_{3}\right)$.

Corollary 5. Let $b^{(1)}$ and $\theta$ be the $5 \times 5$ matrices over $\mathbb{F}_{3}$ given above, and let $\bar{\Omega}^{(1)}, \hat{\Omega}^{(1)}$ be the sets of 242 and 2662 matrices, respectively, defined above. Let $\bar{\Omega}^{(2)}$ and $\hat{\Omega}^{(2)}$ be the sets of matrices obtained by raising every matrix in $\bar{\Omega}^{(1)}$, respectively $\hat{\Omega}^{(1)}$, to the third power.

Then $\bar{\Omega}^{(1)}$ and $\bar{\Omega}^{(2)}$ are two symmetric sets of 242 generators of $\operatorname{PSL}_{5}\left(\mathbb{F}_{3}\right)$, which define isospectral non-isomorphic Cayley complexes. Likewise $\hat{\Omega}^{(1)}$ and $\hat{\Omega}^{(2)}$ are symmetric sets of 2662 generators with the same properties. 
Notice that $\operatorname{PSL}_{5}\left(\mathbb{F}_{3}\right)$ is a group of size $\approx 2.3 \cdot 10^{11}$. Therefore, storing its Cayley graphs in a computer is a difficult task, and computing the eigenvalues directly is nearly impossible.

More generally, we can carry out a similar construction whenever $q$ and $d$ are odd and co-prime, and $e=1$. Following the above mentioned algorithm, take $\beta=1$, then $y(\lambda)=(1+\lambda)^{d}-1$. Take $\alpha=-2$, then $\gamma=y(\alpha)=-2$ and the minimal polynomials are $p(\lambda)=g(\lambda)=\lambda+2$. With $s=1$ we then have $\bar{L}=\mathbb{F}_{q}$ and $x=-2$. Now let $\varphi_{1}$ denote the matrix representing the Frobenius automorphism (in any chosen basis of $\mathbb{F}_{q^{d}}$ over $\left.\mathbb{F}_{q}\right)$. For every $i=1, \ldots, d-1$, let $z_{i}=(1+\beta x) \varphi_{1}^{i}=-\varphi_{1}^{i}$, and $b^{(i)}=1-z_{i}^{-1}=1+\varphi_{1}^{-i}$. Finally fix a generator $\theta$ of $\mathbb{F}_{q^{d}}^{\times} / \mathbb{F}_{q}^{\times}$, and define the sets $\bar{\Omega}^{(i)}$ and $\hat{\Omega}^{(i)}$ as above. Notice that $\left(b^{(i)}\right)^{q}=1+\varphi_{1}^{-q i}=$ $b^{(q i)}$ where the upper index here is modulo $d$, and so the elements of $\Omega^{(q i)}$ are obtained by exponentiating the elements of $\Omega(i)$ to the power $q$.

Corollary 6. Let $q, d$ be odd and co-prime, and let $m$ denote the order of $q$ in $\mathbb{Z} / d \mathbb{Z}^{\times} /\langle \pm 1\rangle$. Let $\varphi_{1}$ denote the Frobenius automorphism of $\mathbb{F}_{q^{d}} / \mathbb{F}_{q}$, and let $\theta$ be an element of $\mathbb{F}_{q^{d}}$ which generates $\mathbb{F}_{q^{d}}^{\times} / \mathbb{F}_{q}^{\times}$. Let $\bar{\Omega}^{(1)}$ be the set of $2 \frac{q^{d}-1}{q-1}$ elements of $\operatorname{PSL}_{d}\left(\mathbb{F}_{q}\right)$ defined as above. Let $\bar{\Omega}^{\left(q^{i}\right)}$ denote the set obtained from $\hat{\Omega}^{(1)}$ by element-wise exponentiation to the power $q^{i}$, for $i=1, \ldots, m-1$.

Then the Cayley graphs of $\mathrm{PSL}_{d}\left(\mathbb{F}_{q}\right)$ with respect to $\bar{\Omega}^{\left(q^{i}\right)}$ are isospectral (even as complexes) and non-isomorphic.

A similar result holds for the sets $\hat{\Omega}^{\left(q^{i}\right)}$.

\section{REFERENCES}

[B] L. Babai, Spectra of Cayley graphs, J. Combin. Theory Ser. B 27(2), 180-189, (1979).

[Br] R. Brooks, Non-Sunada graphs, Ann. Inst. Fourier 49, 707-725, (1999).

[CS] D.I. Cartwright and T. Steger, A family of $\tilde{A}_{n}$-groups, Israel J. Math. 103, 125-140, (1998).

[Jac] N. Jacobson, Finite Dimensional Division Algebras over Fields, Springer, 1996.

[L] F.T. Leighton, Finite common coverings of graphs, J. Comb. Theory (Ser. B) 33, 231-238, (1982). 
[Lu] A. Lubotzky, Cayley graphs: eigenvalues, expanders and random walks, in: Surveys in Combinatorics, London Math. Soc. Lecture Notes Ser. 218, Cambridge University Press, P. Rowlinson (ed.), 155-189, (1995).

[LSV1] A. Lubotzky, B. Samuels and U. Vishne, Ramanujan complexes of type $\tilde{A}_{d}$, Israel J. of Math., to appear.

[LSV2] A. Lubotzky, B. Samuels and U. Vishne, Explicit constructions of Ramanujan complexes, European J. of Combinatorics, to appear.

[LSV3] A. Lubotzky, B. Samuels and U. Vishne, Division algebras and noncommensurable isospectral manifolds, preprint.

[Pe] H. Pesce, Quelques applications de la théorie des représentations en géométrie spectrale, Rend. Mat. Appl. (7) 18(1), 1-63, (1998).

[Pi] R.S. Pierce, Associative Algebras, Graduate Texts in Mathematics 88, 1982.

[R] M. Ronan, Lectures on Buildings, Academic Press, 1989.

[Se] J.J. Seidel, Strongly regular graphs of $L_{2}$-type and of triangular type, Proc. Con. Nederl. Akad. Wetensch. Ser. A 70, 188-196, (1967).

[Su] T. Sunada, Riemannian coverings and isospectral manifolds, Ann. of Math. 121, 169-186, (1985).

[T] J. Tits, Buildings of Spherical Type and Finite BN-Pairs, Lecture Notes in Math. 386, Springer-Verlag.1974

Inst. of Math., Hebrew Univ., Givat Ram, Jerusalem 91904, Israel

E-mail address: alexlub@math.huji.ac.il

Dept. of Math., Yale University, 10 Hillhouse Av., New-Haven CT 06520, USA

E-mail address: beth.samuels@yale.edu

Dept. of Math., Bar-Ilan University, Ramat-Gan 52900, Israel

E-mail address: vishne@math.biu.ac.il 